Vietnam Journal of Mechanics, VAST, Vol.28, No.4 (2006), pp. 207-218

\title{
CALCULATION OF LOADING-INDUCED TENDON SLIP IN BEAMS PRESTRESSED WITH EXTERNAL TENDONS \\ PART II: NUMERICAL ANALYSIS
}

\author{
Bui KHac Diep and Lam HuU Quang \\ Department of Bridge \& Tunnel Engineering, \\ Institute of Transport Science E Technology
}

\begin{abstract}
In this paper, a computing method for the tendon slip based on the force equilibrium condition at deviators was proposed. The proposed method for the evaluation of tendon slip was presented in a general form by taking into account all possibilities of tendon slip at deviators, and several factors found to be important that affect the tendon slip, such as effects of the slippage at two adjacent deviators, friction, change of tendon angles during the applied load, etc. The validation of the proposed method was verified by comparing with the experimental results. The predicted results showed a close agreement with the experimental observation.
\end{abstract}

\section{INTRODUCTION}

The benefit of using the external prestressing had already been proved not only the strengthening of the existing bridges, but also the construction of new bridges. This indicates by a huge number of bridge constructions that have been built by using the external prestressing technique over the world in recent years. The world-wide spread of external prestressing leads to the results of ease construction, time-saving and costeffective bridges thanks to its inherited characteristics from the early days of prestressing development. However, as the external tendons are unbonded with concrete beams except having contact at the deviator points, the stress variation in the external tendons under the applied load depends totally on the deformation of whole member rather than that of particular section itself. Moreover, the stress variation in the external tendons also depends on the extent of fixation of the external tendons at deviators, i.e., it depends on the frictional resistance that exists normally at the contact points between the external tendons and concrete beam. Due to the frictional resistance at deviators, the stress increase in each tendon segment under the applied load will have the effects from one to another between tendon segments. Consequently, it will have the effects on the overall behavior of beam, including the deflection, as well as the ultimate strength of beams.

When beams with deviated tendon profile are subjected to bending, the tensile force in each tendon segment might be different because of the frictional resistance at the deviators. However, when the frictional resistance at a particular deviator is less than the unbalanced force at that deviator, the slip will occur and will continuously occur until the force equilibrium condition at that deviator is achieved to establish a new balanced structural state of tensile forces in the tendon. That is the redistribution of tensile forces between two successive tendon segments will happen through the slippage. Due to the slippage, the 
frictional resistance reduces significantly as the applied load reaches closely the ultimate stage. As a result, the difference in tensile force between two successive tendon segments at ultimate might be rather small, and in some cases the stress in the external tendons could be considered to distribute uniformly over the entire length of tendons.

Although a large number of studies on the behavior of externally prestressed concrete beams were carried out in the past, most of them did not consider the slip at the deviators. Virlogeux [1] was the first author, who took the slip into account in his method of analysis on the basis of Corley's formulation for calculation of the frictional effects. Later, several researchers, for instance [2], [3], [4] and recently [5] took the slip at deviators into account in their methods of analysis. In their methods of analysis, while some of them considered the effects of slip at two adjacent deviators in the slipping zone, the others dealt only with beams having symmetrical arrangement of deviators from the midspan and the symmetrical loading condition. Even though dedicated efforts for the computational method of tendon slip at the deviators had been made in the limited research works, the effect of slip on the behavior of beams prestressed with external tendons still needs to be clearly understood in the depth by means of not only the experimental observation, but also of the analytical investigation. This study is one of the continuous efforts to propose a new computing method for prediction of loading-induced tendon slip at deviators in beams prestressed with external tendons. The validation of the proposed method that was verified by comparing with the experimental results, showed the fitness of the proposed method.

\section{LITERATURE REVIEW}

Herein, only the relevant models to the current research were briefly reviewed in order to have a general view of the computing methods for the tendon slip.

Virloguex [1] was one of the earlier authors, who took slip into account by using Corley's formulation for the calculation of friction losses in the tendons having continuous contact with the concrete along its length. For the author's model, the main restriction for the tendon slip was presented as follows

$$
F_{i} e^{-\left(\mu \Delta \alpha_{i}+\phi \Delta x_{i}\right)} \leq F_{i+1} \leq F_{i} e^{\left(\mu \Delta \alpha_{i}+\phi \Delta x_{i}\right)}
$$

where $F_{i}, F_{i+1}$ are the tendon forces in segments $(i)$ and $(i+1)$, respectively; $\Delta \alpha_{i}$ is the angular variation; $\Delta x_{i}$ is the length of deviation block; $\mu$ and $\phi$ are the friction coefficient and the wobble coefficient per unit length of tendon, respectively.

If these relations are satisfied at any deviator, there is no slip at this deviator. Otherwise, if these relations are not respected for a single deviator, the tendon will slip at this deviator from the left to the right-hand side, if this condition is satisfied:

$$
F_{i+1}>F_{i} e^{\left[\mu \Delta \alpha_{i}+\phi \Delta x_{i}\right]}
$$

and from the right to the left-hand side, if this condition is satisfied:

$$
F_{i+1}<F_{i} e^{\left(\mu \Delta \alpha_{i}+\phi \Delta x_{i}\right)}
$$

If $g_{i}, k_{i}$ are denoted as the amount of tendon slip and the coefficient of slipping direction at deviator ( $i)$, respectively. Depending on the slipping direction, the coefficient of $k_{i}$ may have a value $(-1)$ or $(+1)$ and after slipping, the equilibrium condition should be satisfied the following equation:

$$
F_{i+1}\left(g_{i}\right)=F_{i}\left(g_{i}\right) e^{k_{i}\left(\mu \Delta \alpha_{i}+\phi \Delta x_{i}\right)}
$$


whara $F_{i}\left(g_{i}\right), F_{i+1}\left(a_{i}\right)$ are the tensile forces in tendon segments $(i)$ and $(i+1)$ after the slip with the amount of $g_{i}$, respectively.

In the general case of several simultaneous slips, the tendon slip can be determined as follows

$$
[D]\{\delta g\}=\{P\}
$$

where $[D]$ is the stiffness matrix of slip; $\{\delta g\}$ is the vector of tendon slip; $\{F\}$ is the vector of the tensile forces in the tendons.

Later, Ariyawardena and Ghali [5] used the same condition in Eq. (2.1) for the evaluation of tendon slip at deviators. Due to the slip, the forces in the tendon segments of the other side of deviator are adjusted to the tensile forces in each tendon segment before the slip:

$$
\begin{gathered}
F_{i}^{\prime}=F_{i}-\left(\frac{A_{p s} E_{p s}}{l_{i}}\right) g_{i}, \quad F_{i+1}^{\prime}=F_{i+1}+\left(\frac{A_{p s} E_{p s}}{l_{i+1}}\right) g_{i}, \\
F_{i+1}^{\prime}=F_{i}^{\prime} e^{k_{i}\left(\mu \Delta \alpha_{i}+\phi \Delta x_{i}\right)}
\end{gathered}
$$

where $F_{i}, F_{i+1}$ and $F_{i}^{\prime}, F_{i+1}^{\prime}$ are the tensile forces in segments $(i)$ and $(i+1)$, respectively just before and after the slip at deviators; $A_{p s}$ and $E_{p s}$ are the area and the elastic modulus of tendon; $l_{i}, l_{i+1}$ are the length of tendon segments $(i)$ and $(i+1)$, respectively; $g_{i}$ is the amount of tendon slip at deviator $(i)$.

By solving Eq. (2.6), the trial values of $g_{i}$, the tensile forces after slip can be obtained by using the Newton-Raphson iterative techniques to eliminate the out-of balance forces. The total slip at the deviator can be determined by the sum of slip, $g_{i}$ calculated at each iteration cycle.

Rezende-Martins et al. [2] and Kawamura and Ohura [3] performed nonlinear analysis for externally prestressed beams considering slip at deviators. The basic idea for the tendon slip is conceptually the same as Virlogeux's idea. The only difference is that instead of using the tendon forces in the above formulation, the tendon stress has been used in their formulations. The effect of slip at two adjacent deviators was also taken into account. The formulation for calculation of the tendon slip can be expressed as follows

$$
\sigma_{i} e^{-\mu \Delta \alpha_{i}} \leq \sigma_{i+1} \leq \sigma_{i} e^{\mu \Delta \alpha_{i}}
$$

The equilibrium condition after slip is expressed in terms of the tendon stress with consideration of the slipping direction $k_{i}$ was calculated as follows

$$
\left(\sigma_{i}+\Delta \sigma_{i}\right)=\left(\sigma_{i-1}+\Delta \sigma_{i-1}\right) e^{k_{i} \mu \Delta \alpha_{i}}
$$

The additional increments of tendon stress due to slip can be defined in the following equations:

$$
\begin{aligned}
\left(\sigma_{i}+E_{p s} \frac{g_{i+1}-g_{i}}{l_{i}}\right) & =\left(\sigma_{i-1}+E_{p s} \frac{g_{i}-g_{i-1}}{l_{i-1}}\right) e^{k_{i} \mu \Delta \alpha_{i}} \\
\Delta \sigma_{i-1} & =E_{p s} \frac{g_{i}-g_{i-1}}{l_{i-1}}
\end{aligned}
$$

where $\sigma_{i-1}, \sigma_{i}$ are the tendon stresses in segments $(i-1)$ and $(i)$ before slip; correspondingly, $\Delta \sigma_{i-1}, \Delta \sigma_{i}$ are the additional increments of tendon stress due to slip; $g_{i-1}, g_{i}$ and $g_{i+1}$ are the amounts of tendon slip at deviator $(i-1)$, deviator $(i)$ and deviator $(i+1)$, respectively. 
Finally, the equilibrium condition after tendon slip can be rewritten as:

$$
\begin{gathered}
\left(\sigma_{i}+E_{p s} \frac{g_{i+1}-g_{i}}{l_{i}}\right)=\left(\sigma_{i-1}+E_{p s} \frac{g_{i}-g_{i-1}}{l_{i-1}}\right) e^{k_{i} \mu \Delta \alpha_{i}} \\
\frac{E_{p s} e^{k_{i} \mu \Delta \alpha_{i}}}{l_{i-1}} g_{i-1}-\left(\frac{E_{p s} e^{k_{i} \mu \Delta \alpha_{i}}}{l_{i-1}}+\frac{E_{p s}}{l_{i}}\right) g_{i}+\frac{E_{p s}}{l_{i}} g_{i+1}=\sigma_{i-1} e^{k_{i} \mu \Delta \alpha_{i}}-\sigma_{i}
\end{gathered}
$$

And Eq. (11) can be written in a matrix form as:

$$
[A]\{\delta g\}=\{B\}
$$

where $[A]$ is the stiffness matrix of tendon slip; $\{\delta g\}$ is the vector of tendon slip; $\{B\}$ is the vector of tendon stress.

Rao and Mathew [4] developed a program for nonlinear analysis of externally prestressed concrete beams with polygonal-shaped tendon profile. In the author's methodology, all possibilities of tendon slip at the deviators were taken into account, namely: 1) slip; 2) no slip; 3) no slip early, but slip occurring latter; and 4) early slip, but no slip occurring latter. The author proposed that at any deviator, a slip would occur only if the unbalanced force exceeds the frictional force at that deviator. The unbalanced force can be written as the sum of the frictional force and the additional force that caused the slip at the deviator. For the calculation of tendon slip, the authors proposed the equation as follows

$$
\delta g_{i}=\left[\frac{\mu_{i}\left(F_{i}+F_{i+1}\right) \sin \theta_{i}}{\cos \theta_{i}}\right]\left(\frac{l_{i}}{A_{p s} E_{s p}}\right)
$$

where $A_{p s}, E_{p s}$ are the area and the elastic modulus of prestressing tendons, respectively; $\mu_{i}$ is the friction coefficient at deviator $(i) ; l_{i}$ is the length of tendon segment $(i) ; \theta_{i}$ is the tendon angle; $F_{i}, F_{i+1}$ are the tendon forces at the both sides of deviator $(i)$; the component of $\mu_{i}\left(F_{i}+F_{i+1}\right) \sin \theta_{i}$ in Eq. (2.13) accounts for the frictional force at deviator (i).

It can be seen that Eq. (2.13) seems to be very simple. However, it did not consider the effect of slip at two adjacent deviators. The additional force, which causes slip at a particular deviator, was assumed to be equal to the component of frictional resistance at that deviator, i.e., the actual friction forces before slip were not separated during the calculation of the tendon slip. In addition, the proposed method for calculation of the tendon slip deals only with the beams having symmetrical arrangement of deviators from the midspan and the symmetrical loading condition.

\section{PROPOSED METHOD FOR COM-PUTING TENDON SLIP}

\subsection{Force equilibrium at deviators}

Figure 1 shows that $F_{i}, F_{i+1}$ are the tensile forces in the tendon segments $(i)$ and $(i+1)$ at deviator $(i)$. Correspondingly, $\theta_{i}, \theta_{i+1}$ are the tendon angles, respectively. Thus, the force equilibrium in the $X$ direction can be expressed as follows

$$
F_{i} \cos \theta_{i}+(-1)^{k_{i}} \mu_{i}\left(F_{i} \sin \theta_{i}+F_{i+1} \sin \theta_{i+1}\right)=F_{i+1} \cos \theta_{i+1}
$$

where the coefficient $k_{i}$ depends on the slipping direction, and has a value $k_{i}=1$ if $F_{i} \cos \theta_{i}>F_{i+1} \cos \theta_{i+1}$ and $k_{i}=2$ if $F_{i} \cos \theta_{i}<F_{i+1} \cos \theta_{i+1} ; \mu_{i}$ is the friction coefficient at deviator $(i)$. 
Eq. (3.1) can be rewritten in terms of the increments of tensile forces as follows:

$$
\Delta F_{i} \cos \theta_{i}+(-1)^{k_{i}} \mu_{i}\left(\Delta F_{i} \sin \theta_{i}+\Delta F_{i+1} \sin \theta_{i+1}\right)=\Delta F_{i+1} \cos \theta_{i+1}
$$

where $\Delta F_{i}, \Delta F_{i+1}$ are the increments of tensile forces of tendon at the both sides of the deviator.

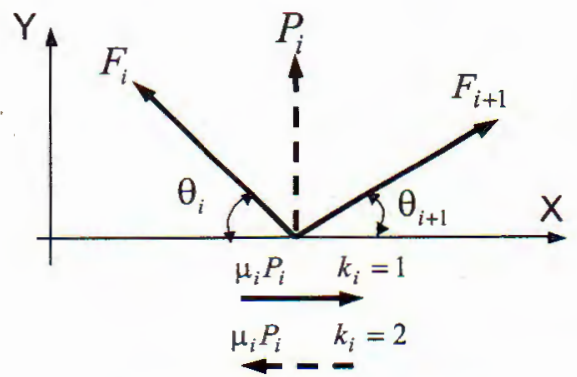

Fig. 1. Force equilibrium at a deviator

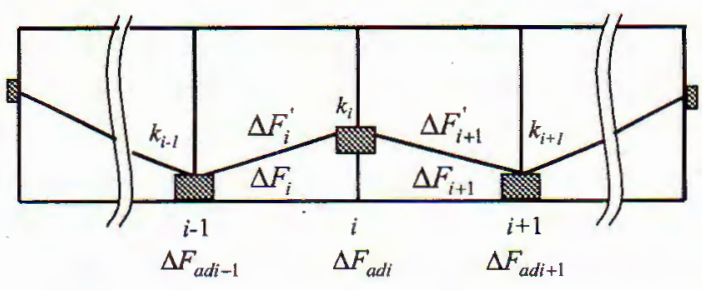

Fig. 2. Equilibrium condition at the slipping zone

\subsection{Criteria for tendon slip}

It is important to note that the tendon slip is not reversible [2]. This means that once the tendon slip occurs at deviator $(i)$, for example from deviator $(i)$ toward deviator $(i+1)$, the slip will continuously occur following this direction until no further slip at this deviator occurs. The change in the direction of the tendon slip will never take place. Any slip that already occurred at any deviator would not reverse, even if the beam was totally unloaded [1]. This was verified by the tested results of box girder beams with external tendons [6].

From Eq. (3.2), the force equilibrium at the deviator can be again rearranged as:

$$
\begin{gathered}
\Delta F_{i+1} \cos \theta_{i+1}-\Delta F_{i} \cos \theta_{i}=(-1)^{k_{i}} \mu_{i}\left(\Delta F_{i} \sin \theta_{i}+\Delta F_{i+1} \sin \theta_{i+1}\right), \\
\Delta F_{\text {dvi }}=\Delta F_{\text {fri }}
\end{gathered}
$$

where $\Delta F_{\text {dvi }}, \Delta F_{\text {fri }}$ are called as the increment of the unbalanced force or the driving force and the increment of the friction force at deviator $(i)$, respectively. They are defined as follows:

$$
\begin{gathered}
\Delta F_{\mathrm{dvi}}=\Delta F_{i+1} \cos \theta_{i+1}-\Delta F_{i} \cos \theta_{i} \\
\Delta F_{\mathrm{fri}}=(-1)^{k_{i}} \mu_{i}\left(\Delta F_{i} \sin \theta_{i}+\Delta F_{i+1} \sin \theta_{i+1}\right)
\end{gathered}
$$

If the increment of the driving force is not equal to zero $\left(\Delta F_{d v i} \neq 0\right)$, Eq. (3.3) can be then written as:

$$
\frac{\Delta F_{\text {fri }}}{\Delta F_{\text {dvi }}}=\lambda
$$

Now, at any loading stage, there are three possibilities: 1) if $\lambda>1$, i.e., the increment of the friction force is greater than the increment of the driving force, the slip cannot occur; 2) if $\lambda=1$ i.e., the increment of the friction force is equal to the increment of the driving force, leading to the force equilibrium condition at a deviator; and 3) if $\lambda<1$, i.e., the increment of the friction force is less than the increment of the driving force, the slip will occur.

From the above condition, at any deviator, the tendon slip will occur only if the increment of the friction force is less than the increment of the driving force. It should 
be noted that the increment of the friction force and the increment of the driving force should be absolute values.

\subsection{Proposed method for tendon slip}

At any loading stage, when the slip occurs at a particular deviator, i.e., $\lambda<1$, the slip will occur continuously until the force equilibrium at that deviator after the slip is achieved. The redistribution of the tendon forces on the both sides of the deviator is allowed through the slippage. An amount of the slip at a particular deviator depends not only on the additional force of this deviator, but also on the additional forces of two adjacent deviators, i.e., one is from the left-hand side and the other is from the right-hand side. It should be noted that the additional force is considered to be a force that causes a slip at a particular deviator at any loading stage. Note that $\Delta F_{i}, \Delta F_{i+1}$ and $\Delta F_{i}^{\prime}, \Delta F_{i+1}^{\prime}$ are denoted as the increments of tendon forces at the either side of deviator just before and after the slip, respectively. The increments of the tendon force after the slip are assumed to be equal to the sum of the increment of the tendon force before the slip plus or minus the actually additional forces of $\Delta F_{\text {ad, }}$, which cause the slip at the deviators (see Fig.2), and these can be expressed as follows:

$$
\begin{gathered}
\Delta F_{i}^{\prime}=\Delta F_{i}+(-1)^{k_{i}} \Delta F_{a d i}+(-1)^{k_{i-1}} \Delta F_{a d i-1} \\
\Delta F_{i+1}^{\prime}=\Delta F_{i+1}+(-1)^{k_{i}+1} \Delta F_{a d i}+(-1)^{k_{i+1}} \Delta F_{a d i+1}
\end{gathered}
$$

The sign of the additional force depends on the slipping direction, i.e., it depends on the coefficient of $k_{i}$. After the slip, a new balanced structural state will be established, being independent of any stiffness change due to material plasticity or cracking along the beam. Therefore, the force equilibrium at the deviator should be satisfied, and can be expressed as follows:

$$
\Delta F_{i}^{\prime} \cos \theta_{i}+(-1)^{k_{i}} \mu_{i}\left(\Delta F_{i}^{\prime} \sin \theta_{i}+\Delta F_{i+1}^{\prime} \sin \theta_{i+1}\right)=\Delta F_{i+1}^{\prime} \cos \theta_{i+1}
$$

Substituting Eq. (3.5) and Eq. (3.6) into Eq. (3.7), after making some manipulations, then it can be obtained as follows

$$
A_{i} \Delta F_{\text {adi-1 }}+B_{i} \Delta F_{a d i}+C_{i} \Delta F_{a d i+1}=\Delta F_{d v i}-\Delta F_{f r i}
$$

where coefficients of $A_{i}, B_{i}$ and $C_{i}$ are defined as follows:

$$
\begin{aligned}
A_{i} & =\left[(-1)^{k_{i-1}}\left(\cos \theta_{i}+(-1)^{k_{i}} \mu_{i} \sin \theta_{i}\right)\right], \\
B_{i} & =\left[\mu_{i}\left(\sin \theta_{i}-\sin \theta_{i+1}\right)+(-1)^{k_{i}}\left(\cos \theta_{i}+\cos \theta_{i+1}\right)\right], \\
C_{i} & =\left[(-1)^{k_{i+1}}\left(-\cos \theta_{i+1}+(-1)^{k_{i}} \mu_{i} \sin \theta_{i+1}\right)\right]
\end{aligned}
$$

For a special case, when the tendon cannot slip at two adjacent deviators, i.e., the additional forces at these deviators are equal to zero, $\left(\Delta F_{\text {adi }-1}=\Delta F_{\text {adi }+1}=0\right)$, Eq. (3.8) can be rewritten as follows:

$$
B_{i} \Delta F_{\text {adi }}=\Delta F_{\text {dvi }}-\Delta F_{\text {fri }}
$$

For the case when the tendon cannot slip at one between two adjacent deviators, there are two possibilities. For the first case, if the tendon did not slip at the left-hand side deviator, the additional force at this deviator is equal to zero, $\Delta F_{\text {adi }-1}=0$. For the second case, if the tendon did not slip at the right-hand side deviator, then $\Delta F_{\text {adi }+1}=0$. 
Therefore, Eq. (3.8) can be rewritten for the two cases in Eq. (3.10) and Eq. (3.11), respectively.

$$
\begin{aligned}
& B_{i} \Delta F_{\text {adi }}+C_{i} \Delta F_{\text {adi }+1}=\Delta F_{\text {dvi }}-\Delta F_{\text {fri }} \\
& A_{i} \Delta F_{\text {adi }-1}+B_{i} \Delta F_{\text {adi }}=\Delta F_{\text {dvi }}-\Delta F_{\text {fri }}
\end{aligned}
$$

To satisfy the global equilibrium condition at the slipping zone, the additional forces at the deviators are obtained by incorporating Eq. (3.8), Eq. (3.10) and Eq. (3.11) in a matrix form with consideration of Eq. (3.9) when it is necessary, and expressed as follows:

$$
\begin{gathered}
{\left[\begin{array}{lllllll}
B_{1} & C_{1} & 0 & \ldots & 0 & 0 & 0 \\
A_{2} & B_{2} & C_{2} & \ldots & 0 & 0 & 0 \\
\cdots & \cdots & \cdots & \ldots & \cdots & \cdots & \cdots \\
\cdots & \cdots & \cdots & B_{i} & \cdots & \cdots & \cdots \\
\cdots & \cdots & \cdots & \cdots & \cdots & \cdots & \cdots \\
0 & 0 & 0 & \cdots & A_{n-1} & B_{n-1} & C_{n-1} \\
0 & 0 & 0 & \ldots & 0 & A_{n} & B_{n}
\end{array}\right]\left\{\begin{array}{l}
\Delta F_{a d 1} \\
\Delta F_{a d 2} \\
\cdots \\
\Delta F_{a d i} \\
\cdots \\
\Delta F_{a d n-1} \\
\Delta F_{a d n}
\end{array}\right\}=\left\{\begin{array}{l}
\Delta F_{d v 1}-\Delta F_{f r 1} \\
\Delta F_{d v 2}-\Delta F_{f r 2} \\
\cdots \\
\Delta F_{d v i}-\Delta F_{f r i} \\
\cdots \\
\Delta F_{d v n-1}-\Delta F_{f r n-1} \\
\Delta F_{d v n}-\Delta F_{f r n}
\end{array}\right\}} \\
{[S]\left\{\Delta F_{a d}\right\}=\{T\}}
\end{gathered}
$$

Finally, the additional forces, which cause a slip at the deviator, are defined as follows

$$
\left\{\Delta F_{a d}\right\}=[S]^{-1}\{T\}
$$

Once the additional force at each deviator is obtained by Eq. (3.13) the amount of tendon slip at each deviator based on the slipping direction, can be determined. The tendon slips from the right to the left-hand side of deviator with $k_{i}=1$ is calculated as follows

$$
\delta g_{i}=\frac{\Delta F_{a d i}}{A_{p s} E_{p s}} l_{i}
$$

The tendon slips from the left to the right-hand side of deviator with $k_{i}=2$ is calculated as follows

$$
\delta g_{i}=\frac{\Delta F_{a d i}}{A_{p s} E_{p s}} l_{i+1}
$$

where $E_{p s}, A_{p s}$ are the elastic modulus and the area of prestressing tendons; $l_{i}, l_{i+1}$ are the tendon lengths of segment $(i)$ and $(i+1)$, respectively; $\delta g_{i}$ is the increment of tendon slip at deviator $(i)$.

Therefore, a possibility for the tendon slip at any deviator depends on the inclined angle of tendon, the unbalanced force in the tendon segments and the friction coefficient at the deviator. For every loading step, according to the deformed shape of a beam, the inclined angle of tendon will change. As a result of the deformed shape of a beam, the change of tendon angle should be considered when the tendon slip is being computed. It should be noted that the proposed equation for the tendon slip is absolutely general, and it can be used to investigate any slip at a particular deviator at the certain loading stage. In addition, the method is applied for the beams with any tendon configuration and number of deviators. 


\section{ACCURACY OF PROPOSED METHOD}

To validate of the proposed method, a computer program for the nonlinear analysis of externally prestressed concrete beams had been developed [7]. In the computer program, a stepwise finite element analysis together with the displacement control method has been used to trace the nonlinear response of prestressed concrete beams with external tendons. The program is capable of accounting not only for the flexural deformation, but also for the shear deformation, friction at the deviators and different configuration of external tendons (straight or polygonal profile). In the analysis, the beam is represented by a set of beam elements connected together by nodes located at either end. Each node has three degree of freedom, namely horizontal displacement, vertical displacement and rotation. Cross section of the beam is divided into layers, in which each layer might have different materials, but its properties are assumed to be constant over the layer thickness. Based on the effective stress in the external tendons, the concrete strain of each layer for every beam element is determined, and appears to take as the initial condition of the beam. It is important to note that the program accounted for the member-analysis, i.e., the deformation of whole member was taken into account.

To evaluate the deformation and the stress in the external tendons, the global requirements of deformation compatibility between the concrete and tendons are required. For this purpose, it is assumed that the total elongation of a tendon must be equal to the integrated value of concrete deformation at the tendon level. Since the prestressing force is transferred only to the concrete beam through the deviator points and the anchorage ends, the frictional resistance obviously exists at the contacted points, resulting in a different level of strain increase between two successive tendon segments. To equilibrate the tensile forces in the tendon segments at both sides of a deviator, the force equilibrium condition must be satisfied, and can be expressed in terms of Eq. (3.1) (see also in Fig. 1). Combining the force equilibrium condition at the deviator with the global deformation compatibility between the external tendons and the concrete beam, the strain variation, and the stress in the external tendons can be computed. In the other words, by summation of the strain increment in each element along the entire length of the beam, the total change in strain, and the change in stress in the external tendons, can be evaluated. Since the strain variation in the external tendons depends mainly on the overall deformation of the beam and the friction at the deviators, the change of the beam deformation due to the applied load is in the relative change of tendon elongation. The adequate evaluation of tendon strain depends the accuracy of the calculation of concrete strain at the tendon level. Therefore, the concrete beam should be necessarily divided into a large number of short elements by using finite element method. The details of computing method for the strain variation in the external tendons due to the applied load can be referred to the previous study [7].

In the analysis, the stress-strain curve for the concrete in compression is assumed to be a parabolic ascending branch and a linear descending branch as shown in Fig. 3. The stress-strain curve is expressed as follows:

$$
\begin{gathered}
f_{c}=f_{c}^{\prime}\left[\frac{2 \varepsilon_{c}}{\varepsilon_{c o}}-\left(\frac{\varepsilon_{c}}{\varepsilon_{c o}}\right)^{2}\right] \text { for } \varepsilon_{c}<\varepsilon_{c o} \\
f_{c}=f_{c}^{\prime}-m_{1}\left(\varepsilon_{c}-\varepsilon_{c o}\right) \text { for } \varepsilon_{c o}<\varepsilon_{c}<\varepsilon_{c u}
\end{gathered}
$$



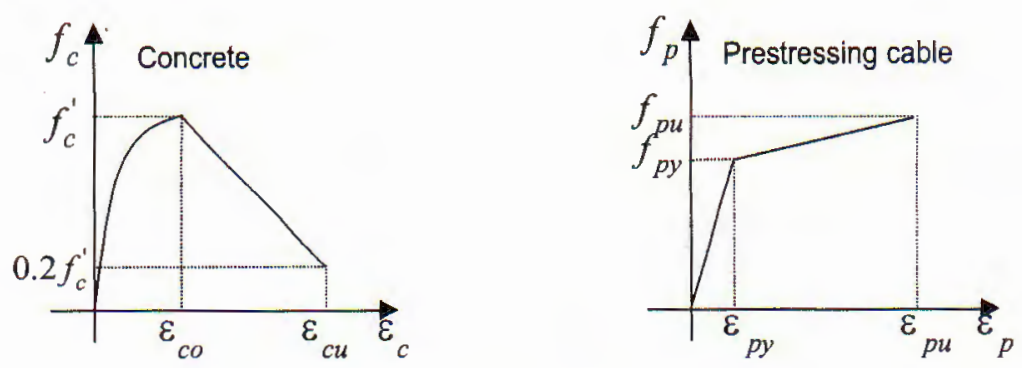

Fig. 3. Stress-strain relationship

where $f_{c}^{\prime}$ is the compressive strength of concrete; $\varepsilon_{c o}=0.002$ is the concrete strain at the peak stress; $\varepsilon_{c u}=0.0035$ is the concrete strain at ultimate; $m_{1}=0.8 f_{c}^{\prime} /\left(\varepsilon_{c u}-\varepsilon_{c o}\right)$ is the post peak slope controlling the descending branch of concrete. A bilinear curve for the stressstrain relationship of prestressing tendons is also presented in Fig. 3 , in which $\varepsilon_{p y}$ and $\varepsilon_{p u}$ has a value of 0.008 and 0.035 , respectively.

To account for the frictional effects, the friction coefficients at deviators were assigned to be 0.15 in consideration of safety factors for all beams. Although this value might not be true in the tested beams, it is, however, only adopted for the analytical purposes. In addition, this value of friction coefficients had been also recommended on the basis of intensive investigation of frictional effects at deviators in beams prestressed with external tendons in the previous study [8].

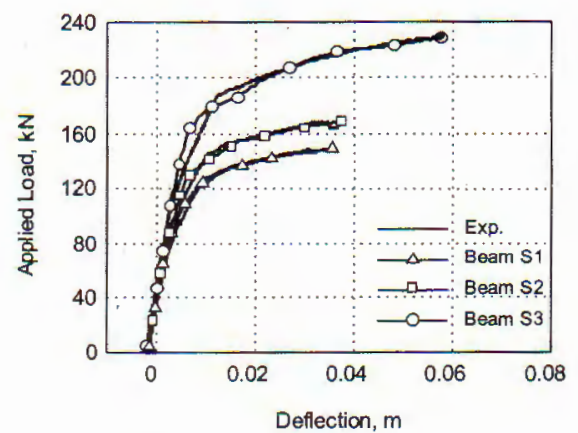

a) Load-deflection curves

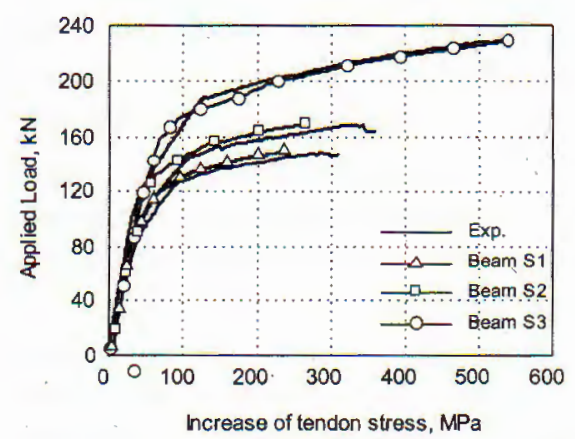

b) Load-stress increase curves

Fig. 4. Comparison with experimental results

Using nonlinear computer program [7] together with the incorporation of proposed method for computing the tendon slip at deviators, a full behavior of externally prestressed concrete beams can be investigated with the emphasis on the effects of slip phenomenon at deviators in the entire loading range. In this study, the behavior of three beams [9] was computed, and predicted results were compared with the experimental observations, as shown in Fig. 4. It is clearly shown that the proposed method of analysis reproduced very accurately the experimental results in terms of both load vs. deflection, and load vs. increase of tendon stress responses over the entire loading range up to failure. Fig. 5 showed the results obtained from the numerical analysis in comparison with the experimental 


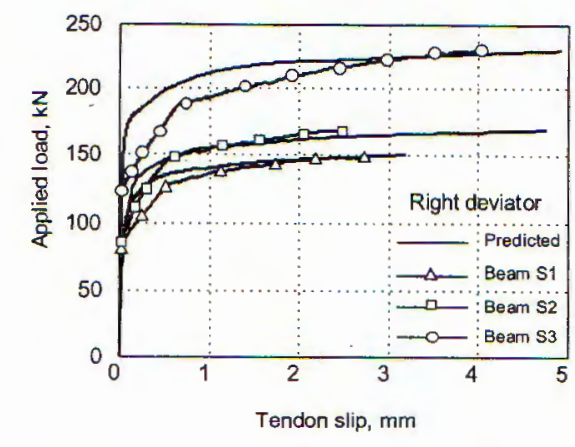

a) Tendon slip at the right deviator

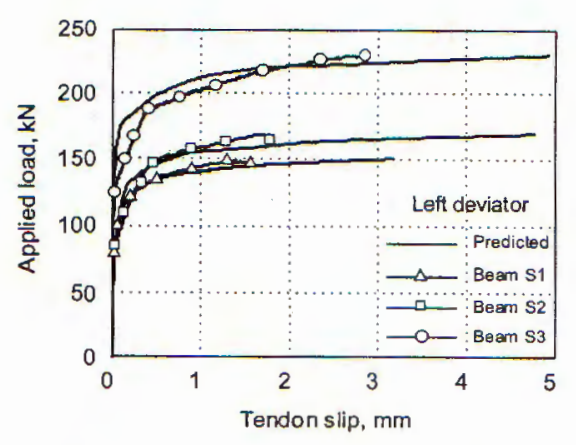

b) Tendon slip at the left deviator

Fig. 5. Comparison of tendon slip with experimental results

observation at both right and left deviators in terms of load-tendon slip relationship. It is observed from this figure that the proposed method for tendon slip, while capturing the experimentally observed trend in the development of tendon slip for all beams, provided the reasonably good agreement in prediction of the tendon slip as compared with the experimental results. It can be seen that the numerical analysis predicted overly the values of tendon slip at both deviators as compared with the experimental results. Larger overestimate of tendon slip at the left deviator was found as compared with those at the right deviator. The overestimate in prediction of the tendon slip can be explained that in the experimental observation, as the tendons began to slip, the frictional resistance at deviators reduced, gradually. Furthermore, the friction coefficient at deviators reduced significantly as the applied load reached closely to the ultimate load. Meanwhile, in the numerical analysis, the friction coefficient at deviators, for the simplified calculation, was kept constantly during the entire loading range. This might lead to the overestimate of tendon slip at deviators as compared with the experimental results. In addition, the tendon slip at deviators is a sophisticated phenomenon, and is very sensitive to any changes in the applied load, the tensile forces in the external tendon, the beam deflection, the frictional resistance at deviators as well as the inclined angles of external tendons, especially at or near by the ultimate state. This can been seen in the results of numerical analysis that all load-tendon slip responses exhibited rather plateaus, i.e., a small change in the applied load made a great change in the tendon slip. Therefore, it is extremely difficult to get "an exact value" of tendon slip at the deviators at ultimate.

Concerning the relations between the stress in the external tendons and the slip phenomenon at deviators, it is evidently indicated that in general the external tendons do not slip from zero-loading until somewhat level of the applied load (approximately 50 60\% of ultimate load for the cases of study) because the frictional resistance at deviators is still big enough to prevent the slip at deviators. This resulted in the different evolution in the stress of each tendon segment. In the other word, the stress increase under the applied load in each tendon segment is independent from each other before slipping. On the other hand, when the slipping load has been reached, tendons began to slip. As a result, the redistribution of stress between tendon segments occurred continuously until no further slipping happened at deviators. It should be noted that the rate of increase in the tendon slip at deviators was higher as the yielding of non-prestressed reinforcements has occurred. 
The larger tendon slip is, the greater rate of redistribution in the stress between tendon segments becomes. This leads to a fairly uniform distribution of the stress in all tendon segments, especially at the ultimate load. Therefore, it is indicated that the increase in the tendon stress under the applied load has a close relationship with the slip phenomenon at: deviators. Finally, through the examples presented in this study, it is able to estimate the influential level of tendon slip at deviators on the stress increase in the external tendons in particular, as well as on the structural behavior of externally prestressed concrete beams in general.

\section{CONCLUSIONS}

This study performed the numerical investigation on the slip phenomenon at deviators in beams prestressed with external tendons. To evaluate the loading-induced tendon slip at deviators, a computer-aided method of analysis was proposed, in which all possibilities of slip and main factors that affect the tendon slip were taken into account. The following aspects concerning the slip phenomenon at deviators in beams prestressed with external tendons can be noted:

(1) The tendon slip at a particular deviator will occur whenever the frictional resistance at that deviator is naturally reduced and is lesser than that of the unbalanced forces between two successive tendon segments. As a result of the slippage, the stress distribution between tendon segments is continuously transferred until the achievement of the force equivalent state at that deviator.

(2) Due to the significant increase in the tendon slip at or near by the peak load, the stress increase in all tendon segments seems to be fairly uniform, especially at the ultimate state.

(3) The tendon slip is a rather complex phenomenon depending on many parameters, and is very sensitive to any change of the structure, material itself and the external factors like the applied load.

It should be noted that the proposed method of analysis, while predicting very accurately the behavior of externally prestressed concrete beams in terms of load-deflection and load-tendon stress curves, provided quite good results in prediction of the tendon slip at deviators as compared with the experimental results. In addition, the computing procedure of tendon slip at deviators should be further improved in order to obtain better results. The evaluation of tendon.slip at deviators in beams prestressed with external tendons.

\section{REFERENCES}

1. M. Virlogeux, Nonlinear analysis of externally prestressed structures, Proceedings of International FIP Symposium, Israel, pp. 319-340, 1988.

2. P. C. Rezende-Martins, P. A. Regis, and J. M. Desir, A study on the behavior of hyperstatic concrete beams with mixed prestressing, Proceedings of the Workshop on Behavior of External Prestressing in Structures, France, pp. 217-226, 1993.

3. N. Kawamura, and T. Ohura, Nonlinear analysis of externally prestressed concrete beams considering friction at deviator, Proceedings of the 5 th Symposium on Development in Prestressed Concrete, pp. 299-302, 1995, (in Japanese). 
4. P. S. Rao, and G. Mathew, Behavior of externally prestressed concrete beams with multiple deviators, ACI Structural Journal 93(4) (1996) 387-396.

5. N. D. Ariyawardena, and A. Ghali, Prestressing with unbonded internal and external tendons: Analysis and Computer Model, ASCE Journal of Structural Engineering 128 (12) (2002) 1493-1501.

6. A. C. Aparicio, G. Ramos, and J. R. Casas, Test of externally prestressed concrete beams, Journal of Engineering Structures, Elsevier Science 24 (1) (2002) 73-84.

7. K. D. Bui, H. Umehara, and T. Tanabe, Numerical analysis of externally prestressed concrete beams considering friction at deviators, JSCE, Journal of Materials, Concrete Structures and Pavements 57 (718) (2002) 107-119.

8. Full-scale test of Kamikazue Bridge - Results of loading test in the prestressing stage, Research Center of Expressway, Oct. 2002.

9. Calculation of loading-induced tendon slip in beams prestressed with external tendons, Part I: Experiment, Vietnam Journal of Mechanics 28 (3) (2006) 165-175.

Received September 07, 2006 Revised September 22, 2006

\section{TÍNH TOÁN SỰ TRƯợT CÁP Ở VẢCH CHUYÊN HƯớNG DƯỚI TÁC DỤNG CỦA TẢI TRỌNG TRONG DẦM BÊ TÔNG CỐT THÉP DỰ ỨNG LỰC NGOÀI PHẦN II: ĐỀ XUẤT PHƯƠNG PHÁP TÍNH TRƯợT CÁP}

Trong bài báo này đề xuất một phương pháp tính toán trượt cáp $\mathrm{CDC}$ trên cơ sở điều kiện cân bằng lực tại các vách chuyển hướng. Phương án đề xuất tính toán trượt cáp được trình bày dưới dạng công thức tổng quát, có xét đến toàn bộ các khả năng trượt cáp có thể xảy ra ờ vách chuyển hướng và một số yếu tố quan trọng ảnh hường đến trượt cáp như ảnh hường của sự trượt cáp ở hai vách chuyển hướng kề cạnh, ma sát, sự thay đổi góc nghiêng của cáp CĐC dưới tác dụng của tải trọng. Độ chính xác của phương pháp tînh toán đề xuất được kiểm chứng thông qua việc so sánh với kết quả thí nghiệm. Các kết quả tính toán dự báo dã chứng minh sự phù hợp tốt với kết quả thí nghiệm. 